\title{
Detection Mass Bias in Atmospheric Pressure Ionization Mass Spectrometry
}

\author{
D. R. Zook and E. P. Grimsrud \\ Department of Chemistry, Montana State University, Bozeman, Montana, USA
}

\begin{abstract}
A previously uncharacterized source of detection mass bias is shown to be associated with atmospheric pressure ionization mass spectrometry (APIMS), and is attributed to a mass dependence in the sampling of ions from the supersonic free jet expansion of gas emerging from the ion source. The halide ions $\mathrm{Cl}^{-}, \mathrm{Br}^{-}$, and $\mathrm{I}^{-}$are shown to be transported from the ion source aperture to a quadrupole mass filter with efficiencies that increase linearly with increasing mass of the ion. While the polyatomic anions $\mathrm{SF}_{6}^{-}$and $\mathrm{C}_{7} \mathrm{~F}_{14}^{-}$are detected with even greater efficiencies than would be expected for monatomic anions of the same mass, this additional sensitivity to the polyatomic anions is thought to be related to ion loss processes occurring within the ion source. The experimental conditions under which these mass bias effects can be minimized or enhanced in APIMS are described. ( $/$ Am Soc Mass Spectrom 1991, 2, 232-239)
\end{abstract}

$\mathrm{I}$ $n$ the use of mass spectrometers for chemical anal$y$ sis and for fundamental studies of gas-phase ion chemistry, knowledge of the variations in the detection sensitivity for ions of differing masses is often required. Sources of detection bias in mass spectrometers are generally associated with the efficiencies of either the mass filter [1] or the ion detector [2]. The acceleration and focusing of ions from an ion source to a mass filter by electric fields should not in general be accompanied by mass bias effects. In this article it will be shown, however, that mass bias effects in the acceleration and focusing region can be extremely important when the mass spectrometer involves aperture sampling of a very high pressure ion source, such as an atmospheric pressure ionization mass spectrometer (APIMS).

In APIMS, the contents of an atmospheric pressure gaseous mixture are passed through an aperture of about $50 \mu \mathrm{m}$ diameter into either one or two adjacent, low pressure regions [3-5]. Because the mean-free path of molecules at atmospheric pressure is much smaller than the dimensions of the aperture, a supersonic free jet expansion is created downstream from the aperture [6, 7]. In a supersonic jet expansion, the carrier gas molecules and all entrained molecules and ions will tend to achieve the same terminal speed and will follow straight stream lines originating near the aperture. 'I he highest intensity of gas flow will be projected along the axis of the aperture. The diffusion of molecules and ions in the directions perpendicular

Address reprint requests to E. P. Grimsrud, Department of Chemistry, Montana State University, Bozeman, MT 59717. to the direction of carrier gas flow is expected to be very slow in an unobstructed jet expansion.

As a first approximation, one might expect that the efficiency with which ions are dirested along the axis of an APIMS ion source jet toward a mass filter and ion detector might be relatively independent of the mass or chemical nature of the ion. However, in previous studies $[8,9]$ of the supersonic jet expansions of neutral binary mixtures, partial separation of the two components of the mixture has been observed at various points in the expansion, generally with enrichment of the more massive component. In the first report of this phenomenon [8], the mechanism was thought to be the preferential radial migration of the lighter species away from the axis of the jet expansion. However, Reis and Fenn [9] later showed that these separation effects in neutral supersonic expansions are primarily caused by the preferential passage of the heavier molecules through a bow shock wave created by placement of a sampling probe within the expansion. In view of these observations, the partial separation of ions of differing mass might also occur in the sampling of a supersonic jet expansion emerging from a high pressure ion source. To our knowledge, however, no quantitative investigations of this type have been reported.

In several previous studies using an APIMS in our laboratory [10-12], we observed mass bias effects that were not thought to be related to the efficiencies of the quadrupole mass filter or to the ion detection system. It was suspected that a significant contribution to mass bias in these studies was associated with the transport of the ions from the ion source aperture 
to a quadrupole mass filter. A systematic characterization of this source of detection bias is reported here, in which the relative collection efficiencies of ions of different mass and chemical composition (atomic versus polyatomic negative ions) have been studied as a function of easily varied physical parameter of an APIMS.

\section{Experimental}

The APIMS ion source and associated ion focusing lenses are shown in Figure 1. The effluent from a gas chromatograph was passed through the ion source at atmospheric pressure with a flow rate of $60 \mathrm{~mL} / \mathrm{min}$. Ionization of the source gas was caused by a $15 \mathrm{mCi}$ ${ }^{63} \mathrm{Ni}$-on-Pt foil, which forms the cylindrical wall of the ion source. About $18 \mathrm{ml} / \mathrm{min}$ of the innized source gas flows through an aperture into the first stage of a differentially pumped vacuum envelope. The aperture (Stork Veco International, Brookline, MA) is made of nickel, has a diameter of $50 \mu \mathrm{m}$ and a depth of $20 \mu \mathrm{m}$, and the shape shown in Figure 1. Three lenses (L1, L2, and L3) focus a portion of the ions emerging from the grounded ion source onto the $2-\mathrm{mm}$ entrance aperture (L4) of a second stage of the vacuum envelope that houses a quadrupole mass filter and ion detector. Unless otherwise indicated, the potentials applied to the four lenses were $+24 \mathrm{~V}(\mathrm{~L} 1),+10 \mathrm{~V}$ (L2), $+24 \mathrm{~V}$ (L3), and $+24 \mathrm{~V}$ (L4). These potentials, with the exception that $\mathrm{L} 2$ be set to $0.0 \mathrm{~V}$, were predicted by the SIMION program [13], to provide optimat focusing. L2 was typically set to $+10 \mathrm{~V}$, however, in order to defocus the ion beam and prevent saturation of the detector. (As will be described in Result, changes in the potential of $\mathbf{L} 2$ have no affect on relative ion intensities.) The potential of L4 is also the reference potential applied to the quadrupole rods. The resolution of the quadrupole mass filter was set very low $(\Delta \mathrm{m} \approx 10 \mathrm{u})$ to ensure efficient transmission

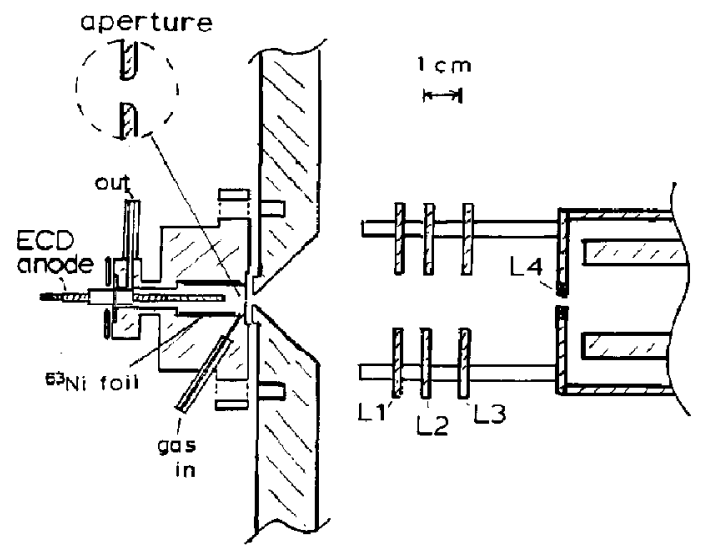

Figure 1. Atmospheric pressure ionization mass spectrometry ion source, ion focusing lenses, and entrance to quadrupole mass filter. of all ions to the ion detector and to allow ions that differ only with respect to isotopic composition to be simultaneously detected. A channeltron ion detector (model 4039, Galileo Electro-Optics Corporation, Sturbridge, MA) was operated in the ion counting mode. The pressures in the first and second vacuum regions were about $7 \times 10^{-4}$ and $7 \times 10^{-6}$ torr, respectively. The ion source was also occasionally operated as a pulsed electron capture detector [10] by the collection of electrons at the anode shown.

The gas chromatograph has a $3 \mathrm{~m} \times 3 \mathrm{~mm}$ column packed with $10 \%$ SF-96 on chromosorb W. Gaseous samples were introduced by a 1-mL sample loop and an associated control valve. These samples were prepared by the successive dilution of the compounds of interest into glass vessels containing nitrogen gas. The carrier gases used were passed through oxygenand water-removing traps prior to the gas chromatograph.

\section{Results}

Gaseous samples containing increasing amounts of either $\mathrm{CCl}_{4}, \mathrm{CH}_{2} \mathrm{Br}_{2}, \mathrm{CH}_{3} \mathrm{I}, \mathrm{SF}_{6}$, or $\mathrm{C}_{7} \mathrm{~F}_{14}$ (perfluoromethylcyclohexane) in nitrogen were chromatographically introduced to the APIMS ion source while monitoring the major negative ion produced by electron capture (EC) to each of these molecules. These ions are $\mathrm{Cl}^{-}, \mathrm{Br}^{-}, \mathrm{I}^{-}, \mathrm{SF}_{6}^{-}$, and $\mathrm{C}_{7} \mathrm{~F}_{14}^{-}$, respectively, and constituted at least $90 \%$ of the total negative ions formed by $\mathrm{EC}$ for each of the compounds. For $\mathrm{SF}_{6}$ and $\mathrm{C}_{7} \mathrm{~F}_{14}$, ions of the type $(\mathrm{M}-\mathrm{F})^{-}$were also observed with intensities about $10 \%$ that of the parent $\mathrm{M}^{-}$ions. The negative ion intensities observed in these experiments are shown in Figure 2, where they have been plotted against a term called "Relative $Q$ " for each compound. This term includes the differences in EC rate coefficients among the compounds studied and accounts for the dilution of compounds in the carrier gas by chromatographic broadening. The magnitude of $Q$ is given by:

$$
\mathrm{Q}=\mathrm{C} \times \mathrm{k}_{\mathrm{ec}} / \mathrm{W}
$$

where $C$ is the molar quantity of the compound introduced for each measurement, $k_{e c}$ is the $E C$ rate coefficient of the compound, and $W$ is the chromatographic peak width observed in the low concentration, linear range of response to each compound. Values of $\mathrm{k}_{\mathrm{ec}}$ are $3.7 \times 10^{-1 /} \mathrm{cm}^{3} / \mathrm{sec}$ for $\mathrm{CCl}_{4}$ [14], $1.6 \times 10^{-7}$ for $\mathrm{CH}_{2} \mathrm{Br}_{2}$ [15], $1.8 \times 10^{-7}$ for $\mathrm{CH}_{3} \mathrm{I}[15]$, $4.5 \times 10^{-7}$ for $\mathrm{SF}_{6}[14]$, and $1.3 \times 10^{-7}$ for $\mathrm{C}_{7} \mathrm{~F}_{14}[15]$ at $150{ }^{\circ} \mathrm{C}$. Normalization of the $Q$ values relative to the case of $\mathrm{CCl}_{4}$ provides Relative $\mathrm{Q}$.

In Figure 2, an increase in signal with increase in Relative $Q$ is initially observed with use of small sample sizes for all compounds. Saturation of the APIMS responses to all compounds then occurs as Relative $Q$ reaches and exceeds a value of about 


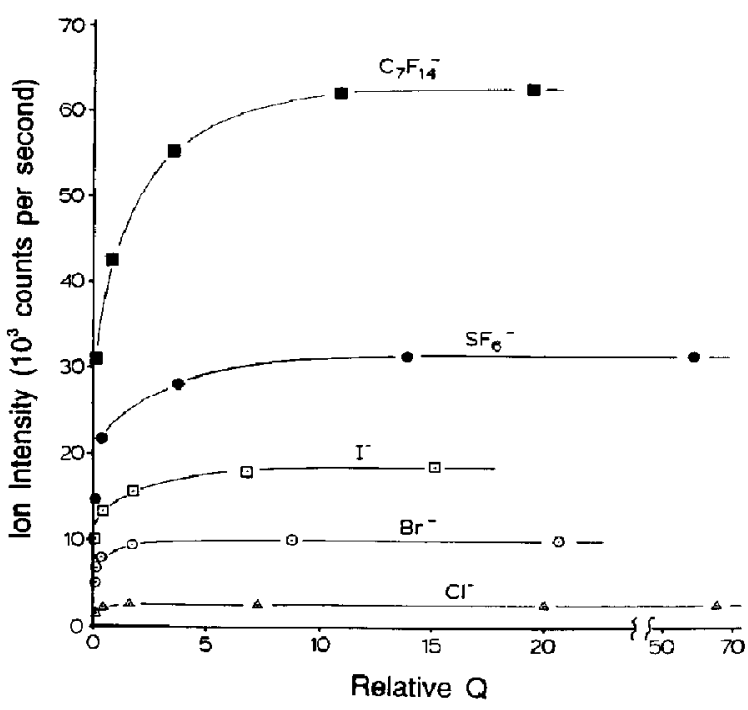

Figure 2. Intensity of the major ion signal observed with the introduction of $\mathrm{CCl}_{4}, \mathrm{CH}_{2} \mathrm{Br}_{2}, \mathrm{CH}_{3} \mathrm{I}, \mathrm{SF}_{6}$, and $\mathrm{C}_{7} \mathrm{~F}_{14}$ to the ion source. Relative $\mathrm{Q}$ is a measure of the relative molar quantity of each compound introduced, corrected for their electron capture coefficients and chromatographic peak widths (1). The carrier gas is nitrogen. The ion source temperature is $150{ }^{\circ} \mathrm{C}$. The potential of $\mathrm{L} 1$ is $+24 \mathrm{~V}$.

$4 \pm 2$. For $\mathrm{CCl}_{4}$, for example, this point corresponds to the introduction of about $80 \mathrm{ng}$ of sample to the ion source. When the ion source shown in Figure 1 was used as an EC detector, analogous points of response saturation were obtained for each compound. These points of EC response saturation are expected to occur when all electrons produced within the ion source have been converted to negative ions by EC reactions. The negative ion production rate is then not changed by the use of higher sample concentrations.

The most significant feature of Figure 2 is that the saturation intensities achieved with higher values of Relative $Q$ differ among the various compounds used. For example, a thirtyfold difference in the saturation levels of the responses to $\mathrm{C}_{7} \mathrm{~F}_{14}$ and $\mathrm{CCl}_{4}$ is observed. From additional experiments not shown in Figure 2, it has also been found that the level of response saturation for a given ion is independent of the compounds used to produce that negative ion. For example, $\mathrm{CCl}_{4}$ and $\mathrm{CHCl}_{3}$ produce the same $\mathrm{Cl}^{-}$ion intensity at saturation even though their EC rate coefficients differ by almost two orders of magnitude [14]. Therefore, the negative ion formed by $\mathrm{EC}$, rather than the parent molecule, determines the saturation level achieved for each case shown in Figure 2.

In Figure 3 saturation responses measured for a set of high concentration samples have been plotted as a function of the masses of the negative ions. We have recently shown [16] that the minor ions of the type $(\mathrm{M}-\mathrm{F})^{-}$, which are observed with relative intensities of about 0.1 for $\mathrm{SF}_{6}$ and $\mathrm{C}_{7} \mathrm{~F}_{14}$, are formed from the

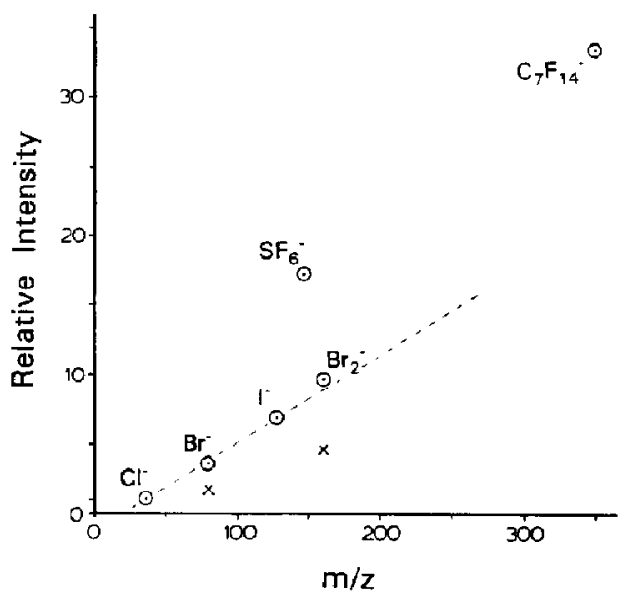

Figure 3. Relative signal intensities observed for saturation level samples of each compound normalized to the case of $\mathrm{CCl}_{4}$. For $\mathrm{SF}_{6}$ and $\mathrm{C}_{7} \mathrm{~F}_{14}$, the indicated intensity of the $\mathrm{M}^{-}$ions also includes the intensities of the minor $(\mathrm{M}-\mathrm{F})^{-}$fragment ions that are simultaneously observed for these two compounds (see text). The carrier gas is nitrogen. The potential of L1 is $+24 \mathrm{~V}$. The dashed line provides the best fit for the monatomic negative ions.

parent $\mathrm{M}^{-}$ions of these molecules by reaction with trace levels of water. If all traces of water could be removed, the intensity of the $(\mathrm{M}-\mathrm{F})^{-}$would decrease to about 0.01 relative abundance and the $\mathrm{M}^{-}$ ion would be correspondingly increased. Therefore, in Figure 3 and in the remaining figures that concern these two compounds, a correction for this loss of a small fraction of the $\mathrm{M}^{-}$ions has been provided by including the intensities of the $(\mathrm{M}-\mathrm{F})^{-}$ions in the indicated $\mathrm{M}^{-}$ion intensities for $\mathrm{SF}_{6}$ and $\mathrm{C}_{7} \mathrm{~F}_{14}$. For the monatomic negative ions $\mathrm{Cl}^{-}, \mathrm{Br}^{-}$, and $\mathrm{I}^{-}$a linear relation exists between the saturation signal intensity and the mass of the ion. It is noted that this line does not pass through the origin of the graph. It is also noted that the data points for the polyatomic anions $\mathrm{SF}_{6}^{-}$and $\mathrm{C}_{7} \mathrm{~F}_{14}^{-}$lie well above the line formed by the monatomic halide anions.

The two data points in Figure 3 indicated by " $X$ " were obtained from experiments performed with $\mathrm{CF}_{3} \mathrm{CFBrCF}_{2} \mathrm{Br}$ in which $\mathrm{Br}^{-}$and $\mathrm{Br}_{2}^{-}$were produced simultaneously in a branched dissociative $\mathrm{EC}$ reaction. The $\mathrm{Br}^{-}$ion signal at saturation is 0.50 as large as the $\mathrm{Br}^{-}$signal produced by $\mathrm{CH}_{2} \mathrm{Br}_{2}$. Therefore, it appears that an equal amount of the two negative ions $\mathrm{Br}^{-}$and $\mathrm{Br}_{2}^{-}$are produced in the $\mathrm{EC}$ of $\mathrm{CF}_{3} \mathrm{CFBrCF}_{2} \mathrm{Br}$ and that the second data point " $\mathrm{X}$ " in Figure 3 indicates the saturation level of a compound that provides $50 \% \mathrm{Br}_{2}^{-}$upon $\mathrm{EC}$. By doubling the magnitude of this data point, the expected relative saturation intensity for a compound that would produce only $\mathrm{Br}_{2}^{-}$upon EC is provided. It is seen in Figure 3 that the relative saturation intensity for $\mathrm{Br}_{2}^{-}$ also lies somewhat above the line determined by the monatomic negative ions. 


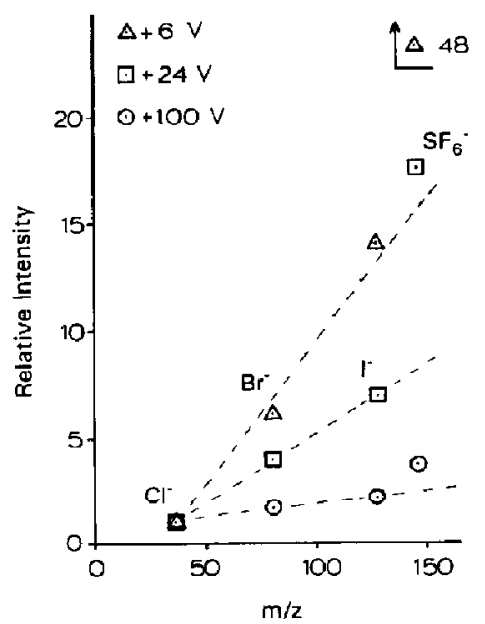

Figure 4. Effect of L1 potential on relative saturation signal intensities. The carrier gas is nitrogen.

The experiments that led to Figure 3 were also performed with different potentials applied to the focusing lenses shown in Figure 1. Varying the potentials of $\mathrm{L} 2$ and $\mathrm{L} 3$ over the range 0 to $+24 \mathrm{~V}$ had no significant effect on the relative ion intensities observed for all compounds. Variations of L4 and the quadrupole potential that is tied to this lens also had no significant effect on relative signals over the range +12 to $+24 \mathrm{~V}$. As expected, the application of offset potentials smaller than $+12 \mathrm{~V}$ to the quadrupole rods caused the progressive loss of sensitivity to the higher mass ions due to the excessively low longitudinal velocities for heavy ions within the quadrupole region under these conditions [1].

In Figure 4, the effect of changing the potential of $\mathrm{L} 1$ from $+24 \mathrm{~V}$ to $+6 \mathrm{~V}$ and to $+100 \mathrm{~V}$ is shown. It is seen that the ion biases previously noted are signifcantly enhanced by use of the lower potential and are greatly reduced by use of the higher potential applied to L1.

In Figure 5, the effect of changing the carrier gas from nitrogen to argon or to methane is shown. Mass bias effects are significantly increased with use of argon and are significantly decreased with use of methane.

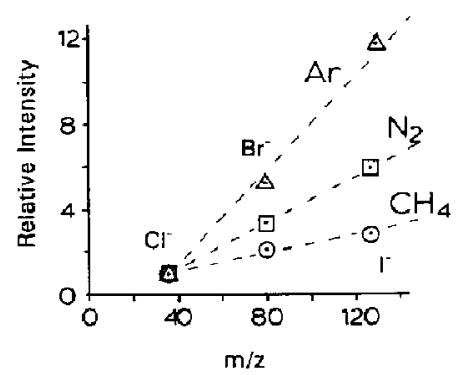

Figure 5. Relative saturation signal intensities using argon, nitrogen, and methane carrier gas. The potential of $L 1$ is $+24 \mathrm{~V}$.

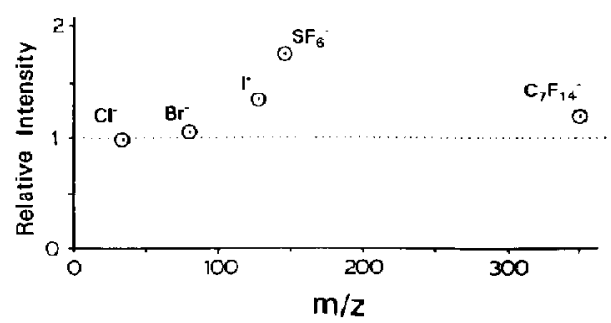

Figure 6. Relative saturation signal intensities using methane carrier gas and an L1 potential of $+100 \mathrm{~V}$.

In Figure 6, the relative saturation signals are shown under a combination of conditions that, based on the above results, should provide minimal overall ion detection bias in the APIMS. These conditions include the use of methane buffer gas and the application of a relatively high potential to $\mathrm{L} 1$. While detection mass bias has not been completely eliminated in Figure 6, it has been reduced to relatively small levels.

\section{Discussion}

In identifying the individual elements that contribute most significantly to the overall detection biases shown in Figures 2-6, five distinct regions of the instrument must be considered. These are the ion source, the free jet expansion region (between the ion source aperture and L1), the focusing region between $\mathrm{L} 1$ and $\mathrm{L} 4$, the quadrupole mass filter, and the detector. From results described above, it is apparent that mass bias effects are either very small or nonexistent in two of these regions, between $\mathrm{L} 1$ and $\mathrm{L} 4$ and in the quadrupole region. Potential contributions to detection bias originating in the remaining three regions (the ion detector, the ion source, and the free jet expansion) will be individually considered below in order of their perceived increasing importance.

\section{Ion Detector}

The ion detector used was of the channeltron type and was operated in the ion counting mode. The detector was mounted on the quadrupole axis directly behind the exit orifice of the quadrupole housing. With this detector and its associated preamplifier and pulse height discriminator, each ion that strikes the cone should provide one count to the rate meter, provided at least one electron is produced upon impact of the negative ion. In this case, the detection system should not contribute to mass or ion-type biases. However, if the efficiency of electron ejection from the cone is less than unity, the arrival of some ions would not be recorded and contributions to mass or ion-type biases by the detection system would then be possible. In an attempt to minimize this potential source of detection bias, we have taken care to ensure 
that the high voltage applied to the channeltron was always well above that needed to reach the response plateau of the multiplier. We also noted that the relationships between the detector's response and applied high voltage, and between the onset of responses and the maximum responses, were essentially identical for all of the ions studied here. In light of these observations, any contributions to mass bias effects by the channeltron detector are thought to be relatively small.

\section{Ion Source}

An important feature in the experimental design of this study is that a well-characterized and highly reproducible ${ }^{63} \mathrm{Ni}$-based ion source has been used for the generation of the negative ions. With this ion source, the relative production and destruction rates of negative ions and, therefore, the steady-state populations of the negative ions are thought to be under relatively good experimental control. The rate of negative ion production at saturation is limited by and equal to the rate of secondary electron production through the interaction of the ${ }^{63} \mathrm{Ni}$ beta radiation with the buffer gas molecules [17]. Therefore, the negative ion production rate is identical for all of the EC-active compounds used here. The dominant means of negative ion loss within an atmospheric pressure ion source of moderately high ion density will be by positive ion-negative ion recombination [18, 19]. At atmospheric pressure ion-ion recombination is a three-body process involving buffer gas molecules as well as the two ionic reagents $[20]$. While three-body ion-ion recombination reactions have not been extensively studied, their rate coefficients are thought to be relatively independent of the identity of the ions involved [20, 21]. If the ion-ion recombination coefficients are, in fact, exactly the same for different ions, the steadystate concentration of negative ions in our samplesaturated ion source is expected to be identical for all of the compounds studied here. In this case, ion source processes could be ruled out entirely as a contributor to the overall differences in the saturation signals observed in Figure 3.

Experimental evidence that events occurring in the ion source are not the major cause of differences in the saturation signals observed in Figure 3 is provided by Figure 4 . Figure 4 shows that the relative saturation intensities are very sensitive to changes in the electric field set between the ion source flange and L1. Due to the small size $(50 \mu \mathrm{m})$ of the electrically grounded inn source aperture, the penetration of the electric field into the interior of the ion source is expected to be very weak and the transport of ions out of the ion source should be determined only by convective flow of the carrier gas through the aperture. Therefore, the fact that differences in the saturation signals in Figure 4 are very sensitive to the applied electric field indicates that the ion biases are being caused primarily outside rather than inside the ion source. Also, the fact that the mass biases are greatest with use of the weakest field (about $1 \mathrm{~V} / \mathrm{cm}$ ) would be very difficult to explain in terms of ion source processes.

Due to inadequate present knowledge concerning ion-ion recombination reactions, however, it must be acknowledged that a minor contribution to the relative saturation signals in Figure 3 might be caused by differences in the rates at which different negative ions are destroyed in the ion source. Such differences would create slightly different steady-state concentrations of negative ions within the source and might cause the residual differences in the saturation signals shown in Figure 6, for example, where the largest contributions to bias have been greatly reduced by use of a high extraction potential and methane carrier gas. The unusually large saturation responses to $\mathrm{SF}_{6}$ and $\mathrm{C}_{7} \mathrm{~F}_{14}$ that have been consistently observed in this study (Figures 3, 4, and 6) might be explained if the polyatomic anions undergo ion-ion recombination somewhat more slowly than the atomic anions, resulting in slightly higher steady state concentrations of the polyatomic anions in the ion source at saturation. This contribution to relative saturation signals would then always be present and would be amplified by additional and larger effects caused outside the ion source, as in Figure 3.

\section{Free Jet Expansion}

Although ion source events may contribute to the differing saturation signals in Figure 3 , it is clear from the experiments reported in Figure 4 that the major contribution to detection bias resides in the region between the ion source aperture and $\mathrm{L} 1$.

One relatively trivial explanation for the differing saturation currents in Figure 3 is that a negative ion space-charge potential in this region causes the preferential migration of lighter ions away from the central axis. However, it can be shown that this possibility is unlikely due to the very low ion currents provided by a ${ }^{63} \mathrm{Ni}$-based ion source $[18,19]$. The total negative ion current entering the first vacuum region is less than $1 \times 10^{8}$ ions $/ \sec (<20 \mathrm{pA})$. The residence time of ions between the aperture and $\mathrm{L} 1$ will be less than $20 \mu \mathrm{s}$ (assuming that the minimum ion velocity is equal to the flow velocity of the jet). Therefore, even if all of the negative ions were confined to a relatively small cylindrical volume of radius $0.3 \mathrm{~cm}$ on the axis between the ion source aperture and L1, the density of negative ions in this volume would be less than $2 \times 10^{3}$ ions/cc. Application of Poisson's equation [22] indicates that the space-charge electric fields would be less than $1 \mathrm{mV} / \mathrm{cm}$ at all points within this volume. The magnitude of this space-charge field is clearly insignificant relative to that of the externally applied electric field determined by the potential of L1. 
Because the more familiar causes of detection mass bias discussed above do not adequately account for the large mass bias effects shown in Figure 3, it is concluded they are caused by less well-understood processes associated with the supersonic free jet expansion and the electrostatic ion sampling device. In the following discussion of this possibility, it is useful to describe first the expected location of the supersonic free jet expansion relative to the position of the focusing elements shown in Figure 1.

The so-called silent zone [23] of the gas expansion, where molecules tend to move with equal speed in the same direction, will extend out to the "mach disk, " a transition region at which directed flow of the expanding gas changes to normal random motion. The length of the silent zone is expected to be approximately equal to 0.67 times the diameter of the sampling aperture times the square root of the ratio of the pressures in the ion source and first vacuum envelope regions [23]. For our instrument, this distance is predicted to be about $3 \mathrm{~cm}$ from the aperture, which is also the location of the first focusing L1 shown in Figure 1. If the electric field induced by $\mathrm{L1}$ is momentarily ignored, all ions entrained in the silent zone would be expected to attain the same directed velocity of the bulk gas, and their relative kinetic energies would then be in proportion to their masses. For example, in a supersonic jet expansion of nitrogen gas from a high pressure reservoir at $150^{\circ} \mathrm{C}, \mathrm{Cl}^{-}$and $\mathrm{C}_{7} \mathrm{~F}_{14}^{-}$ions entrained in this jet would be expected to achieve kinetic energies of approximately $0.17 \mathrm{eV}$ and $1.6 \mathrm{eV}$, respectively [24].

The accelerating effect of the electric field must also be considered, however. The ion sampling system shown in Figure 1 attempts to electrostatically focus the ions emerging from the ion source onto the entrance aperture of the quadrupole housing. The ions in the jet region will be accelerated by the field established by $\mathrm{L1}(+24 \mathrm{~V}$ in Figure 3), and may attain kinetic energies $(24 \mathrm{eV})$ that are considerably greater then the energies imparted by the directed flow of the jet. In that case the approximately 1.4-eV energy difference between the $\mathrm{Cl}^{-}$and $\mathrm{C}_{7} \mathrm{~F}_{14}^{-}$ions provided by the nitrogen jet expansion would be relatively insignificant. However, in at least the initial portions of the jet, where gas density is very high, collisions will significantly retard the acceleration of ions through the electric freld and the ions will acquire only a portion of the full kinetic energy potentially available from the extraction field. Therefore, the jet-induced differences in the kinetic energies may be significant relative to the total average energies of the ions as they approach L1.

We have considered the possibility that these nonuniform kinetic energies cause the mass bias effect in Figure 3 merely through a dependence of ion energy on the efficiency of ion focusing onto the entrance aperture of the quadrupole. In this case, it should be possible to focus preferentially ions of any selected energy range onto the entrance aperture of the quadrupole housing. However, this possibility is not supported by the observations made here that the detection mass biases were always in favor of the higher masses (i.e., the higher energy ions) and were quantitatively unchanged by alterations in the potentials of $\mathrm{L} 2$ and $\mathrm{L} 3$.

A more satisfactory explanation appears to be one based on that provided by Reis and Fenn [9] in their investigations of the expansions of neutral gaseous mixtures. They concluded that the heavier molecules entrained in a jet expansion will preferentially penetrate through a bow shock wave that is set up by the presence of a sampling cone. In our case, if a bow shock wave is established in front of L1, it would tend to direct the flow of molecules and entrained ions away from the central axis and would inhibit their passage through L1. The passage of ions and neutrals through this shock wave would be assisted by their increased energy. Increased energy would be attained for heavy ions versus light ions by entrainment in the jet. This factor is thought to explain the major portion of the detection biases shown in Figure 3. Increased energy is also imparted to all ions by use of higher electric fields in the region of the bow shock wave. This is thought to explain the results shown in Figure 4.

The results shown in Figure 5, where different buffer gases have been used, might also be adequately explained in terms of the transport of ions through the bow shock wave along with the so-called velocity slip effect [?] of free jet expansions. It is well known that entrained molecules will not always achieve the high speed of the jet if the difference in the masses of the seeded compunuls and the cutrrier gas molecules is sufficiently large. Velocity slip has been shown to be significant not only in helium, but also in neon [25], which has greater mass than methane. It may be reasonable, therefore, to suggest that velocity slip is operative for at least methane carrier gas in Figure 5. Velocity slip would be more extensive for the more massive $\mathrm{I}^{-}$ion than for $\mathrm{Cl}^{-}$ ion and, therefore, the $\mathbf{I}^{-}$ions would gain a smaller fraction of the full velocity of the methane jet. This would explain the reduction of mass bias effects for methane in Figure 5, and would also be consistent with the increased mass bias effects observed in argon buffer gas.

\section{Conclusions}

The experiments reported here demonstrate that the relative signal intensities observed in APIMS can be subject to large mass bias effects that are associated with the supersonic free jet expansion of gas emerging from the ion source and the sampling of the entrained ions by electrostatic lenses. These effects are presently thought to be due to the preferential penetration of heavier ions through a bow shock wave 
A

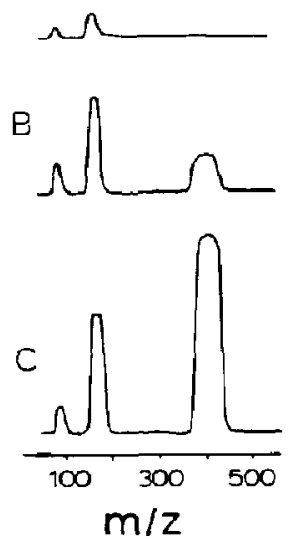

Figure 7. Low-resolution atmospheric pressure ionization mass spectrometry negative ion spectra of 1,2-dibromoperfluoropropane taken (a) at the leading edge of the chromatographic peak, (b) a few seconds later, and (c) at the center of the chromatographic peak. The carrier gas is nitrogen, The ion source temperature is $100^{\circ} \mathrm{C}$. The concentration of the sample is 3 ppth (mole ratio). The potential of L1 is $+24 \mathrm{~V}$.

set up by the first ion focusing lens. If desired, these mass bias effects can be greatly reduced by the use of methane rather than nitrogen carrier gas, and by the use of relatively high electric fields in the region of the jet expansion. While the very light carrier gases hydrogen and helium were not included in this study because of their low beta-attenuating ability in a ${ }^{63} \mathrm{Ni}$ ion source [26], a further reduction of mass bias effects in APIMS might be expected with their use. Conversely, one can easily envision applications in chemical analysis by APIMS in which large ion bias effects would be desirable for the selective analysis of large ions. Therefore, it is equally important to note that mass bias effects were significantly enhanced with use of argon rather than nitrogen buffer gas and by use of very low extraction fields. The magnitude of jet-induced mass bias effects in APIMS will undoubtedly also depend on additional physical factors not explored here, such as the size and shape of the ion source aperture and the size, shape, and location of the ion sampling device [9].

In the use of an APIMS ion source for fundamental studies of gas-phase ion chemistry, the determination of and correction for mass bias effects may often be necessary. An illustration of this point is provided in Figure 7, where three very low resolution negative ion mass spectra of 1,2-dibromoperfluoropropane (DBPFP) are shown during the gas chromatographic introduction of a relatively large amount of this compound to our APIMS ion source. Spectrum A was taken at the leading edge of the chromatographic peak when the concentration of DBPFP was relatively low. Only the two $\mathrm{EC}$ products $\mathrm{Br}^{-}$and $\mathrm{Br}_{2}^{-}$are observed. While these two ions are actually formed in approximately equal amounts [12], the intensity of the $\mathrm{Br}_{2}^{-}$signal is about twice as great as the $\mathrm{Br}^{-}$signal due to mass bias. Spectrum $B$ was taken moments later when the concentration of DBPFP in the ion source was greater. An additional ion of relatively high mass is then observed due to the clustering equilibrium reaction $\mathrm{Br}^{-}+$DBPFP $=\mathrm{Br}^{-}$(DBPFP), It is noted that no cluster ion of the type $\mathrm{Br}_{2}^{-}$(DBPFP) is observed. Spectrum $C$ was taken at the center of the chromatographic peak when the concentration of DBPFP was greatest; the intensity of the high mass cluster ion is then again increased. A particularly deceptive feature of these spectra, however, is that while the cluster ion grows from almost zero intensity in spectrum $A$ to dominance in spectrum $\mathrm{C}$, the intensity of the $\mathrm{Br}^{-}$signal decreases only slightly relative to that of $\mathrm{Br}_{2}^{-}$, which is not being consumed by a clustering reaction. This dilemma is explained, however, by the fact that the overall detection sensitivity of the APIMS to the cluster ion is about ten times greater than to the $\mathrm{Br}^{-}$ion under the experimental conditions used here and, therefore, the apparent importance of the cluster ion is greatly exaggerated. While detection biases could be reduced for this system by the use of higher electric fields, this remedy might introduce an even greater problem by facilitating the collisional decomposition of the cluster ions in the expansion region.

\section{Acknowledgment}

This work was supported by grant from the Chemical Analysis Division of the National Science Foundation (grant no. CHE8711618) and the Montana/EPSCoR II Program of the National Science Foundation (grant no. R11-8921978).

\section{References}

1. Watson, J. T. Introduction to Mass Spectrometry; Raven Press: New York, 1985; p. 88.

2. Stafford, G. C.; Reeher, J. R.; Story, R. S. In Mass Spectrometry, Part B; Merritt , C.; McEwen, C. N., Eds.; Marcel Dekker: New York, 1990; p. 359.

3. Horning, E. C.; Horning, M. G.; Carroll, D. I.; Dzidic, I.; Stillwell, R. N. Anal. Chem. 1973, 45, 936.

4. Carroll, D. I.; Dzidic, I.; Horning, E. C.; Stillwell, R. N. Appl. Spectros. Rev. 1981, 17, 337.

5. Bruins, A. P. Mass Spec. Rev. 1991, 10, 53.

6. Johnston, M. V. Trends in Anal. Chem. 1984, 3, 58.

7. Hayes, J. M. Chem. Rev. 1987, 87, 745.

8. Stern, S. A.; Waterman, P. C.; Sinclair, T. F. I. Chem, Phys. 1960, 33, 805.

9. Reis, V. H.; Fenn, J. B. J. Chem. Phys. 1963, 39, 3240.

10. Grimsrud, E. P.; Kim, S. H. Anal. Chem. 1979, 51, 537.

11. Mock, R. S.; Zook, D. R.; Grimsrud, E. P. Int. J. of Mass Spec, and Ion Proc. 1989, 91, 327.

12. Zook, D. R.; Knighton, W. B.; Grimsrud, E. P. Int. I. of Mass Spec. and Ion Proc. 1990, in press. 
13. Dahl, D. A.; Delmore, J. E. The SIMION PC/PC2 User's Manual, Version 3.1, a report from of Idaho National Engineering Laboratory; Idaho Falls, Idaho, November 1987.

14. Smith, D.; Adams, N. G.; Alge, E. I. Phys. B: At. Mol. Phys. 1984, 17, 461.

15. Alge, E.; Adams, N. G.; Smith, D. J. Phys. B 1984, 17, 3827.

16. Knighton, W. B.; Zook, D. R.; Grimsrud, E. P. J. Am. Soc. Mass Spectrom. 1990, 1, 372.

17. Gobby, P. L.; Grimsrud, E. P.; Warden, S. W. Anal. Chem. $1980,52,473$.
18. Siegel, M. W.; Mckeown, M. C. J. Chromatogr. 1976, 122, 397.

19. Siegel, M. W.; Fite, W. L. J. Phys. Chem. 1976, 80, 2871

20. Smith, D.; Church, M. J. Planet. Space Sci. 1977, 25, 433.

21. Mock, R. S.; Grimsrud, E. P. Anal. Chem. 1988, 60, 1684.

22. Lorrain, P.; Corson D. Electromagnetic Fields and Waves, 2nd ed.; W. H. Freeman and Co.: San Francisco, 1970; p. 51.

23. Campargue, N. I. Phys. Chem, 1984, 88, 4466.

24. Hagena, O. F. Surface Sci. 1981, 106, 101.

25. Amirav, A.; Even, U.; Jortner, J. Chem. Phys. 1980, 51, 31.

26. Grimsrud, E,; Connolly, M. J. J. Chromatogr. 1982, 239, 397. 